\title{
A Rotation of Admixable Operators on Abstract Wiener Space with Applications
}

\author{
Jae Gil Choi and Seung Jun Chang \\ Department of Mathematics, Dankook University, Cheonan 330-714, Republic of Korea \\ Correspondence should be addressed to Seung Jun Chang; sejchang@dankook.ac.kr
}

Received 17 December 2012; Accepted 24 June 2013

Academic Editor: Henryk Hudzik

Copyright (C) 2013 J. G. Choi and S. J. Chang. This is an open access article distributed under the Creative Commons Attribution License, which permits unrestricted use, distribution, and reproduction in any medium, provided the original work is properly cited.

We investigate certain rotation properties of the abstract Wiener measure. To determine our rotation property for the Wiener measure, we introduce the concept of an admixable operator via an algebraic structure on abstract Wiener space. As for applications, we define the analytic Fourier-Feynman transform and the convolution product associated with the admixable operators and proceed to establish the relationships between this transform and the corresponding convolution product.

\section{Introduction and Preliminaries}

Let $\left(C_{0}[0, T], \mathscr{M}, m_{w}\right)$ denote one-parameter Wiener space. Bearman's rotation theorem [1] for Wiener measure has played an important role in various research areas in mathematics and physics involving Wiener integration theory. Bearman's theorem was further developed by Cameron and Storvick [2] and by Johnson and Skoug [3] in their studies of Wiener integral equations. Recently, in [4], using results in [5], Chang et al. obtained results involving a very general multiple Fourier-Feynman transform on Wiener space.

Let $H$ be a real separable infinite-dimensional Hilbert space with inner product $\langle\cdot, \cdot\rangle$ and norm $|\cdot|=\sqrt{\langle\cdot, \cdot\rangle}$. Let $\|\cdot\|_{0}$ be a measurable norm on $H$ with respect to the Gaussian cylinder set measure $v_{0}$ on $H$. Let $B$ denote the completion of $H$ with respect to $\|\cdot\|_{0}$. Let $i$ denote the natural injection from $H$ to $B$. The adjoint operator $i^{*}$ of $i$ is one to one and maps $B^{*}$ continuously onto a dense subset of $H^{*}$, where $B^{*}$ and $H^{*}$ are topological duals of $B$ and $H$, respectively. By identifying $H^{*}$ with $H$ and $B^{*}$ with $i^{*} B^{*}$, we have a triple $B^{*} \subset H^{*} \approx H \subset B$ and $\langle x, y\rangle=(x, y)$ for all $x$ in $H$ and $y$ in $B^{*}$, where $(\cdot, \cdot)$ denotes the natural dual pairing between $B$ and $B^{*}$. By the well-known result of Gross [6], $v_{0} \circ i^{-1}$ provides a unique countably additive extension, $\nu$, to the Borel $\sigma$-algebra $\mathscr{B}(B)$ of $B . v$ is a probability measure on the Borel $\sigma$-algebra $\mathscr{B}(B)$ of $B$ which satisfies

$$
\int_{B} \exp \{i(y, x)\} d \nu(x)=\exp \left\{-\frac{1}{2}|y|^{2}\right\} \quad \text { for every } y \in B^{*} \text {. }
$$

The triple $(B, H, \nu)$ is called an abstract Wiener space. For more details, see [6-9].

Let $\left\{e_{j}\right\}_{j=1}^{\infty}$ be a complete orthonormal set in $H$ such that $e_{j}$ 's are in $B^{*}$. For each $h \in H$ and $x \in B$, we define the stochastic inner product $(h, x)^{\sim}$ by

$$
(h, x)^{\sim}= \begin{cases}\lim _{n \rightarrow \infty} \sum_{j=1}^{n}\left\langle h, e_{j}\right\rangle\left(e_{j}, x\right), & \text { if the limit exists, } \\ 0, & \text { otherwise. }\end{cases}
$$

For every $h(\neq 0)$ in $H,(h, x)^{\sim}$ exists for $\nu$-a.e. $x \in B$, and it is a Gaussian random variable on $B$ with mean zero and variance $|h|^{2}$; that is, (1) holds with $y \in B^{*}$ replaced with $h \in H$. In fact, the stochastic inner product $(h, x)^{\sim}$ is essentially independent of the choice of the complete orthonormal set used in its definition. Also, if both $h$ and $x$ are in $H$, then Parseval's 
identity gives $(h, x)^{\sim}=\langle h, x\rangle$. Furthermore, $(h, \lambda x)^{\sim}=$ $(\lambda h, x)^{\sim}=\lambda(h, x)^{\sim}$ for any $\lambda \in \mathbb{R}, h \in H$, and $x \in B$. We also see that, if $\left\{h_{1}, \ldots, h_{n}\right\}$ is an orthonormal set in $H$, then the random variables $\left(h_{j}, x\right)^{\sim}$ 's are independent.

Let $\mathscr{W}(B)$ be the class of $\nu$-measurable subsets of $B$. A subset $E$ of $B$ is said to be scale-invariant measurable $[3,7]$ provided $\rho E$ is $\mathscr{W}(B)$-measurable for every $\rho>0$, and a scale-invariant measurable subset $N$ of $B$ is said to be scaleinvariant null provided $\nu(\rho N)=0$ for every $\rho>0$. A property that holds except on a scale-invariant null set is said to hold scale invariant almost everywhere (s-a.e.). A functional $F$ on $B$ is said to be scale-invariant measurable provided $F$ is defined on a scale-invariant measurable set and $F(\rho \cdot)$ is $\mathscr{W}(B)$-measurable for every $\rho>0$. If two functionals $F$ and $G$ on $B$ are equal $s$-a.e., that is, for each $\rho>0, \nu(\{x \in B$ : $F(\rho x) \neq G(\rho x)\})=0$, then we write $F \approx G$.

Next, we introduce the concept of an admixable operator on $B$.

Definition 1. Let $\odot$ be an operation between $H$ and $B^{*}$ which satisfies the conditions:

(1) $B^{*} \times B^{*} \ni\left(g_{1}, g_{2}\right) \mapsto g_{1} \odot g_{2}=g_{2} \odot g_{1} \in B^{*}$.

(2) $H \times B^{*} \ni(h, g) \mapsto h \odot g=g \odot h \in H$.

(3) If $h \odot g=0$ for $h \in H$ and $g \in B^{*}$, then $h=0$ or $g=0$.

(4) For every $h \in H$ and every $g_{1}, g_{2} \in B^{*}$,

$$
\left(h \odot g_{1}\right) \odot g_{2}=h \odot\left(g_{1} \odot g_{2}\right) .
$$

(5) For every $h_{1}, h_{2} \in H$ and every $g \in B^{*}$,

$$
\left(h_{1}+h_{2}\right) \odot g=h_{1} \odot g+h_{2} \odot g .
$$

(6) For every $g_{1}, g_{2} \in B^{*}$, there exists $g_{3} \in B^{*}$ such that

$$
g_{1}^{\odot 2}+g_{2}^{\odot 2}=g_{3}^{\odot 2}
$$

where $g^{\odot 2}=g \odot g$. In this case, we write $g_{3}=$ $\sqrt[\odot]{g_{1}^{\odot 2}+g_{2}^{\odot 2}}$.

(7) For every $h_{1}, h_{2} \in H$ and every $g \in B^{*}$,

$$
\left\langle h_{1}, h_{2} \odot g\right\rangle=\left\langle h_{1} \odot g, h_{2}\right\rangle .
$$

Given $g \in B^{*}$, let $A_{g}: B \rightarrow B$ be a linear operator associated with $g$. The operator $A_{g}$ is said to be $g^{\odot}$-admixable provided $\left(h, A_{g} x\right)^{\sim}=(h \odot g, x)^{\sim}$ for all $h \in H$.

For a finite subset $\mathscr{V}=\left\{v_{1}, \ldots, v_{m}\right\}$ of $H$, let $X_{\mathscr{V}}: B \rightarrow$ $\mathbb{R}^{m}$ be the random vector given by

$$
X_{\mathscr{V}}(x)=\left(\left(v_{1}, x\right)^{\sim}, \ldots,\left(v_{m}, x\right)^{\sim}\right) \equiv(\vec{v}, x)^{\sim} .
$$

A functional $F$ is called a cylinder-type functional on $B$ if there exists a linearly independent subset $\mathscr{V}=\left\{v_{1}, \ldots, v_{m}\right\}$ of $H$ such that

$$
F(x)=\psi\left(X_{\mathscr{V}}(x)\right), \quad x \in B,
$$

where $\psi$ is a complex-valued Lebesgue measurable function on $\mathbb{R}^{m}$. It is easy to show that, for the given cylinder-type functional $F$ of the form (8), there exists an orthogonal subset $\mathscr{H}=\left\{h_{1}, \ldots, h_{n}\right\}$ of $H$ such that $F$ is expressed as

$$
F(x) \equiv f\left(X_{\mathscr{H}}(x)\right)=f\left(\left(h_{1}, x\right)^{\sim}, \ldots,\left(h_{n}, x\right)^{\sim}\right), \quad x \in B,
$$

where $f$ is a complex-valued Lebesgue measurable function on $\mathbb{R}^{n}$. Thus, we lose no generality in assuming that every cylinder-type functional on $B$ is of the form (9).

Lemma 2 (Chung, [7]). Let $(B, H, v)$ be an abstract Wiener space, and let $\mathscr{H}=\left\{h_{1}, \ldots, h_{n}\right\}$ be an orthogonal set in $H$. Let $f$ be a complex-valued function defined on $\mathbb{R}^{n}$. Then, for the cylinder-type functional $F$ given by (9) on $B$,

(i) $F$ is $\mathscr{B}(B)$-measurable if and only if $f$ is Borel measurable on $\mathbb{R}^{n}$,

(ii) $F$ is $\mathscr{W}(B)$-measurable if and only if $f$ is Lebesgue measurable on $\mathbb{R}^{n}$.

For $g \in B^{*}$, let $A_{g}$ be the $g^{\odot}$-admixable operator on $B$. In this case, for any orthogonal subset $\mathscr{H}$ of $H$,

$$
\begin{aligned}
X_{\mathscr{H}}\left(A_{g} x\right) & =\left(\left(h_{1}, A_{g} x\right)^{\sim}, \ldots,\left(h_{n}, A_{g} x\right)^{\sim}\right) \\
& =\left(\left(h_{1} \odot g, x\right)^{\sim}, \ldots,\left(h_{n} \odot g, x\right)^{\sim}\right) .
\end{aligned}
$$

The seminal results by Bearman in [1] are summarized as follows (see [2]): if $F\left(\sqrt{a^{2}+b^{2}} x\right)$ is Wiener integrable on $C_{0}[0, T]$ for $a, b \in \mathbb{R}$, then $F\left(a x_{1}+b x_{2}\right)$ is integrable on $\left(C_{0}[0, T]\right)^{2}$ and

$$
\begin{gathered}
\int_{\left(C_{0}[0, T]\right)^{2}} F\left(a x_{1}+b x_{2}\right) d\left(m_{w} \times m_{w}\right)\left(x_{1}, x_{2}\right) \\
=\int_{C_{0}[0, T]} F\left(\sqrt{a^{2}+b^{2}} x\right) d m_{w}(x) .
\end{gathered}
$$

The main purpose of this paper is to establish a rotation property for the abstract Wiener integral,

$$
\begin{aligned}
\int_{B^{2}} F & \left(A_{g_{1}} x_{1}+A_{g_{2}} x_{2}\right) d(\nu \times v)\left(x_{1}, x_{2}\right) \\
& =\int_{B} F\left(A_{g} x\right) d \nu(x),
\end{aligned}
$$

where $F$ is given by (9) and $A_{g}$ is determined by $A_{g_{1}}$ and $A_{g_{2}}$.

\section{A Typical Example of an Abstract Wiener Space}

The classical Wiener space $C_{0}[0, T]$, which is one of the most important examples of abstract Wiener spaces (see [9]), is a triple $(B, H, \nu)$, where

(i) $B=C_{0}[0, T]$ is a Banach space consisting of realvalued continuous functions $x(t)$ with $x(0)=0$ defined on the compact interval $[0, T]$ endowed with uniform norm $\|x\|=\sup _{t \in[0, T]}|x(t)|$, 
(ii) $H=C_{0}^{\prime}[0, T]$ is a real separable infinite dimensional Hilbert space consisting of absolutely continuous functions $h(t)$ with $h(0)=0$, such that $D h \equiv d h / d t \in$ $L_{2}[0, T]$ endowed with the inner product

$$
\left\langle h_{1}, h_{2}\right\rangle=\int_{0}^{T} D h_{1}(s) D h_{2}(s) d s,
$$

(iii) $v=m_{w}$ is the Wiener measure on the Borel $\sigma$-algebra $\mathscr{B}\left(C_{0}[0, T]\right)$ of $C_{0}[0, T]$ with

$$
m_{w}(\{x: x(t) \leq a\})=\int_{-\infty}^{a} \exp \left\{-\frac{u^{2}}{2 t}\right\} d u .
$$

Let $I$ be the unitary operator from $L_{2}[0, T]$, onto $C_{0}^{\prime}[0, T]$ given by $I v(t)=\int_{0}^{t} v(s) d s$ for $v \in L_{2}[0, T]$ and let

$$
\begin{gathered}
C_{0}^{*}[0, T]=\{I v: v \text { is continuous except for a finite } \\
\text { number of finite jump discontinuities } \\
\text { and is of bounded variation on }[0, T]\} .
\end{gathered}
$$

For any $h \in C_{0}^{\prime}[0, T]$ and $g \in C_{0}^{*}[0, T]$, let the operation $\odot$ between $C_{0}^{\prime}[0, T]$ and $C_{0}^{*}[0, T]$ be defined by

$$
h \odot g=I(D h D g) \text {, }
$$

where $D h D g$ denotes the pointwise multiplication of the functions $\mathrm{Dh}$ and $\mathrm{Dg}$.

It is readily seen that $\left\{x(t):(x, t) \in C_{0}[0, T] \times[0, T]\right\}$ is a standard Wiener process on the probability space $\left(C_{0}[0, T], \mathscr{B}\left(C_{0}[0, T]\right), m_{w}\right)$. In this case, we know that, for each $h \in C_{0}^{\prime}[0, T]$ and $s$-a.e. $x \in C_{0}[0, T]$,

$$
(h, x)^{\sim}=\int_{0}^{T} D h(t) \tilde{d} x(t),
$$

where $\int_{0}^{T} D h(t) \widetilde{d} x(t)$ denotes the Paley-Wiener-Zygmund stochastic integral [10-12].

Remark 3. Let $M$ be the set of all $m_{w}$-measurable subsets of $C_{0}[0, T]$. Then, $\left(C_{0}[0, T], \mathscr{M}, m_{w}\right)$ is a complete measure space. It is well known that $\mathscr{M}$ coincides with $\sigma\left(\mathscr{B}\left(C_{0}[0, T]\right)\right)$, the completion of $\mathscr{B}\left(C_{0}[0, T]\right)$.

Let $g \in C_{0}^{\prime}[0, T]$ with $|g|=\sqrt{\langle g, g\rangle}>0$. Then, the stochastic integral

$$
\mathscr{Z}_{g}(x, t)=\int_{0}^{t} \operatorname{Dg}(s) \tilde{d} x(s), \quad t \in[0, T],
$$

which was introduced by Park and Skoug in [13], is a Gaussian process with mean zero and covariance function

$$
\int_{C_{0}[0, T]} \mathscr{Z}_{g}(x, s) \mathscr{Z}_{g}(x, t) d m_{w}(x)=\int_{0}^{\min \{s, t\}}(D g(u))^{2} d u .
$$

In addition, $\mathscr{Z}_{g}(x, t)$ is stochastically continuous in $t$ on $[0, T]$. For more detailed studies of this process, see [14-17]. Furthermore, if $g$ is an element of $C_{0}^{*}[0, T]$, then, for all $x \in$ $C_{0}[0, T], \mathscr{Z}_{g}(x, t)$ is continuous in $t$, and so is $\mathscr{Z}_{g}(x, \cdot)$ in $C_{0}[0, T]$.

From [14, Lemma 1], we note that, for each $h \in C_{0}^{\prime}[0, T]$ and each $g \in C_{0}^{\prime}[0, T]$ with $D g \in L_{\infty}[0, T]$,

$$
\int_{0}^{T} D h(t) \tilde{d}_{g}(x, t)=\int_{0}^{T} D h(t) D g(t) \tilde{d} x(t)
$$

for s-a.e. $x \in C_{0}[0, T]$.

Given $g \in C_{0}^{*}[0, T]$, define an operator $A_{g}: C_{0}[0, T] \rightarrow$ $C_{0}[0, T]$ by

$$
A_{g} x=\mathscr{Z}_{g}(x, \cdot)
$$

Then, for all $h \in H$,

$$
\begin{aligned}
\left(h, A_{g} x\right)^{\sim} & =\int_{0}^{T} D h(t) \tilde{d} \mathscr{Z}_{g}(x, t) \\
& =\int_{0}^{T} D h(t) D g(t) \tilde{d} x(t)=(h \odot g, x)^{\sim} .
\end{aligned}
$$

Thus, $A_{g}$ is $g^{\odot}$-admixable in view of Definition 1 .

\section{A Rotation of Admixable Operators}

In this section, we establish a rotation property for the abstract Wiener integral involving admixable operators. We first introduce an integration formula which plays a key role.

Lemma 4. Let $\mathscr{A}=\left\{\alpha_{1}, \ldots, \alpha_{n}\right\}$ be an orthogonal set in $H$ and let $X_{\mathscr{A}}$ be given by (7). Let $f: \mathbb{R}^{n} \rightarrow \mathbb{C}$ be a Lebesgue measurable function. Then

$$
\begin{aligned}
\int_{B} f & \left(X_{\mathscr{A}}(x)\right) d \nu(x) \\
= & \int_{B} f\left(\left(\alpha_{1}, x\right)^{\sim}, \ldots,\left(\alpha_{n}, x\right)^{\sim}\right) d \nu(x) \\
\stackrel{*}{=}\left(\prod_{j=1}^{n} 2 \pi\left|\alpha_{j}\right|^{2}\right)^{-1 / 2} & \times \int_{\mathbb{R}^{n}} f\left(u_{1}, \ldots, u_{n}\right) \\
& \times \exp \left\{-\sum_{j=1}^{n} \frac{u_{j}^{2}}{2\left|\alpha_{j}\right|^{2}}\right\} d u_{1} \cdots d u_{n},
\end{aligned}
$$

where by $\stackrel{*}{=}$ one means that if either side exists, both sides exist and equality holds.

The following integration formula is also used several times in this paper:

$$
\int_{\mathbb{R}} \exp \left\{-a u^{2}+b u\right\} d u=\sqrt{\frac{\pi}{a}} \exp \left\{\frac{b^{2}}{4 a}\right\}
$$

for complex numbers $a$ and $b$ with $\operatorname{Re}(a)>0$. 
Remark 5. Let $\alpha^{1}$ and $\alpha^{2}$ be elements in $H$ with $\left|\alpha^{1}\right|=\left|\alpha^{2}\right| \equiv$ $\sigma^{2}>0$. Then, the random variables $X_{1}(x)=\left(\alpha^{1}, x\right)^{\sim}$ and $X_{2}(x)=\left(\alpha^{2}, x\right)^{\sim}$ will have the same distribution $N\left(0, \sigma^{2}\right)$.

Let $\mathscr{A}_{1}=\left\{\alpha_{1}^{1}, \ldots, \alpha_{n}^{1}\right\}$ and $\mathscr{A}_{2}=\left\{\alpha_{1}^{2}, \ldots, \alpha_{n}^{2}\right\}$ be orthogonal sets in $H$ with $\left|\alpha_{j}^{1}\right|=\left|\alpha_{j}^{2}\right|$ for each $j \in\{1, \ldots, n\}$. Using the aforementioned facts and applying (23), we see that, for any Lebesgue measurable function $f$ on $\mathbb{R}^{n}$,

$$
\int_{B} f\left(X_{\mathscr{A}_{1}}(x)\right) d \nu(x) \stackrel{*}{=} \int_{B} f\left(X_{\mathscr{A}_{2}}(x)\right) d \nu(x)
$$

To simplify the expressions in our results, we use the following notations:

$$
\begin{gathered}
f(\vec{u}) \equiv f\left(u_{1}, \ldots, u_{n}\right), \\
f\left(\vec{u}+X_{\mathscr{A}}(x)\right) \equiv f\left(u_{1}+\left(\alpha_{1}, x\right)^{\sim}, \ldots, u_{n}+\left(\alpha_{n}, x\right)^{\sim}\right)
\end{gathered}
$$

for $\vec{u}=\left(u_{1}, \ldots, u_{n}\right) \in \mathbb{R}^{n}$ and $X_{\mathscr{A}}(x)$ given by $(7)$.

Proposition 6. Let $\mathscr{A}_{1}=\left\{\alpha_{1}^{1}, \ldots, \alpha_{n}^{1}\right\}$ and $\mathscr{A}_{2}=\left\{\alpha_{1}^{2}, \ldots, \alpha_{v}^{2}\right\}$ be orthogonal sets in $H$. Then, for any Lebesgue measurable function $f$ on $\mathbb{R}^{n}$,

$$
\begin{aligned}
\int_{B^{2}} f & \left(X_{\mathscr{A}_{1}}\left(x_{1}\right)+X_{\mathscr{A}_{2}}\left(x_{2}\right)\right) d(\nu \times v)\left(x_{1}, x_{2}\right) \\
& \stackrel{*}{=} \int_{B} f\left(X_{\mathscr{W}_{(2)}}(x)\right) d v(x)
\end{aligned}
$$

where $\mathscr{W}_{(2)}=\left\{w_{1}, \ldots, w_{n}\right\}$ is an orthogonal set in $H$ which satisfies the condition

$$
\left|w_{j}\right|^{2}=\left|\alpha_{j}^{1}\right|^{2}+\left|\alpha_{j}^{2}\right|^{2}
$$

for each $j \in\{1, \ldots, n\}$. Also, both of the expressions in (27) are given by the last expression in (29).

Proof. First, using (23), the Fubini theorem, and (24), we have that

$$
\begin{aligned}
& \int_{B^{2}} f\left(X_{\mathscr{A}_{1}}\left(x_{1}\right)+X_{\mathscr{A}_{2}}\left(x_{2}\right)\right) d(\nu \times v)\left(x_{1}, x_{2}\right) \\
&= \int_{B}\left[\int_{B} f\left(X_{\mathscr{A}_{1}}\left(x_{1}\right)+X_{\mathscr{A}_{2}}\left(x_{2}\right)\right) d \nu\left(x_{1}\right)\right] d \nu\left(x_{2}\right) \\
&=\left(\prod_{j=1}^{n} 2 \pi\left|\alpha_{j}^{1}\right|^{2}\right)^{-1 / 2} \\
& \times \int_{\mathbb{R}^{n}}\left[\int_{B} f\left(\vec{u}+X_{\mathscr{A}_{2}}\left(x_{2}\right)\right) d \nu\left(x_{2}\right)\right] \\
& \times \exp \left\{-\sum_{j=1}^{n} \frac{u_{j}^{2}}{2\left|\alpha_{j}^{1}\right|^{2}}\right\} d \vec{u}
\end{aligned}
$$

$$
\begin{gathered}
=\left(\prod_{j=1}^{n} 2 \pi\left|\alpha_{j}^{1}\right|^{2}\right)^{-1 / 2}\left(\prod_{j=1}^{n} 2 \pi\left|\alpha_{j}^{2}\right|^{2}\right)^{-1 / 2} \\
\times \int_{\mathbb{R}^{n}}\left[\int_{\mathbb{R}^{n}} f(\vec{u}+\vec{v})\right. \\
\left.\times \exp \left\{-\sum_{j=1}^{n} \frac{v_{j}^{2}}{2\left|\alpha_{j}^{2}\right|^{2}}\right\} d \vec{v}\right] \\
\times \exp \left\{-\sum_{j=1}^{n} \frac{u_{j}^{2}}{2\left|\alpha_{j}^{1}\right|^{2}}\right\} d \vec{u}
\end{gathered}
$$$$
=\left(\prod_{j=1}^{n} 2 \pi\left|\alpha_{j}^{1}\right|^{2}\right)^{-1 / 2}\left(\prod_{j=1}^{n} 2 \pi\left|\alpha_{j}^{2}\right|^{2}\right)^{-1 / 2}
$$$$
\times \iint_{\mathbb{R}^{n}} f(\vec{r}) \exp \left\{-\sum_{j=1}^{n} \frac{\left(r_{j}-u_{j}\right)^{2}}{2\left|\alpha_{j}^{2}\right|^{2}}\right\}
$$$$
\times \exp \left\{-\sum_{j=1}^{n} \frac{u_{j}^{2}}{2\left|\alpha_{j}^{1}\right|^{2}}\right\} d \vec{u} d \vec{r}
$$

$$
\begin{aligned}
= & \left(\prod_{j=1}^{n} 2 \pi\left|\alpha_{j}^{1}\right|^{2}\right)^{-1 / 2}\left(\prod_{j=1}^{n} 2 \pi\left|\alpha_{j}^{2}\right|^{2}\right)^{-1 / 2} \\
& \times \int_{\mathbb{R}^{n}} f(\vec{r})\left[\int_{\mathbb{R}^{n}} \exp \left\{-\sum_{j=1}^{n} \frac{u_{j}^{2}}{2\left|\alpha_{j}^{1}\right|^{2}}-\sum_{j=1}^{n} \frac{\left(r_{j}-u_{j}\right)^{2}}{2\left|\alpha_{j}^{2}\right|^{2}}\right\} d \vec{u}\right] d \vec{r} \\
= & \left.\left(\prod_{j=1}^{n} 2 \pi\left(\left|\alpha_{j}^{1}\right|^{2}+\left|\alpha_{j}^{2}\right|^{2}\right)\right)^{-1 / 2}\right] \\
& \times \int_{\mathbb{R}^{n}} f(\vec{r}) \exp \left\{-\sum_{j=1}^{n} \frac{r_{j}^{2}\left(\left|\alpha_{j}^{1}\right|^{2}+\left|\alpha_{j}^{2}\right|^{2}\right)}{d \vec{r} .}\right.
\end{aligned}
$$

Next, let $\left\{e_{1}, \ldots, e_{n}\right\}$ be any orthonormal set in $H$. For each $j \in\{1, \ldots, n\}$, let $w_{j}=\left(\left|\alpha_{j}^{1}\right|^{2}+\left|\alpha_{j}^{2}\right|^{2}\right)^{1 / 2} e_{j}$. Then $\mathscr{W}_{(2)}=$ $\left\{w_{1}, \ldots, w_{n}\right\}$ is an orthogonal set in $H$ and satisfies (28) above. In this case, using (23), the Fubini theorem, and (24), we see that $\int_{B} f\left(X_{\mathscr{W}_{(2)}}(x)\right) d \nu(x)$ is given by the last expression of (29). In view of Remark 5, we obtain the desired result.

The following corollary follows by the use of mathematical induction. 
Corollary 7. For each $k \in\{1, \ldots, m\}$, let $\mathscr{A}_{k}=\left\{\alpha_{1}^{k}, \ldots, \alpha_{n}^{k}\right\}$ be an orthogonal set in $H$. Then for any Lebesgue measurable function $f$ on $\mathbb{R}^{n}$,

$$
\begin{aligned}
\int_{B^{m}} f & \left(X_{\mathscr{A}_{1}}\left(x_{1}\right)+\cdots+X_{\mathscr{A}_{m}}\left(x_{m}\right)\right) d \nu^{m}\left(x_{1}, \ldots, x_{m}\right) \\
& \stackrel{*}{=} \int_{B} f\left(X_{\mathscr{W}_{(m)}}(x)\right) d \nu(x)
\end{aligned}
$$

where $\mathscr{W}_{(m)}=\left\{w_{1}, \ldots, w_{n}\right\}$ is an orthogonal set in $H$ which satisfies the condition

$$
\left|w_{j}\right|^{2}=\sum_{k=1}^{m}\left|\alpha_{j}^{k}\right|^{2}
$$

for each $j \in\{1, \ldots, n\}$. Also, both of the expressions in (30) are given by the expression

$$
\begin{aligned}
& \left(\prod_{j=1}^{n} 2 \pi\left(\sum_{k=1}^{m}\left|\alpha_{j}^{k}\right|^{2}\right)\right)^{-1 / 2} \\
& \quad \times \int_{\mathbb{R}^{n}} f(\vec{r}) \exp \left\{-\sum_{j=1}^{n} \frac{r_{j}^{2}}{2\left(\sum_{k=1}^{m}\left|\alpha_{j}^{k}\right|^{2}\right)}\right\} d \vec{r} .
\end{aligned}
$$

The next corollary follows directly from Proposition 6.

Corollary 8. Let $\mathscr{A}_{1}$ and $\mathscr{A}_{2}$ be as in Proposition 6. Assume that $\mathscr{A}_{1} \cup \mathscr{A}_{2}$ is an orthogonal set in $H$. Then, for any Borel measurable function $f$ on $\mathbb{R}^{n},(27)$ is valid. In this case, $\mathscr{W}_{(2)}$ is given by the $\operatorname{set}\left\{\alpha_{1}^{1}+\alpha_{1}^{2}, \ldots, \alpha_{n}^{1}+\alpha_{n}^{2}\right\}$.

Throughout the rest of this section, for convenience, we use the following notation: for a finite sequence $\mathscr{G}=$ $\left\{g_{1}, \ldots, g_{m}\right\}$ in $B^{*}$, let

$$
s(\mathscr{G}) \equiv s\left(g_{1}, \ldots, g_{m}\right)=\sqrt[\odot]{\sum_{k=1}^{m} g_{k}^{\odot 2}}
$$

For our rotation property presented, namely, Theorem 11, we will consider the pair of finite subsets $\mathscr{H}=\left\{h_{1}, \ldots, h_{n}\right\}$ of $H-\{0\}$ and $\mathscr{G}=\left\{g_{1}, \ldots, g_{m}\right\}$ of $B^{*}-\{0\}$ (this allows $|\mathscr{G}| \leq m$ ) such that

(c1) $\left\{h_{1}, \ldots, h_{n}\right\}$ is orthogonal in $H$,

(c2) $\left\{h_{1} \odot g_{k}, \ldots, h_{n} \odot g_{k}\right\}$ is orthogonal in $H$ for all $k \in$ $\{1, \ldots, m\}$.

Let us return to the classical Wiener space $\left(C_{0}[0, T]\right.$, $\left.C_{0}^{\prime}[0, T], m_{w}\right)$. See Section 2. For each $l \in\{1,2, \ldots\}$, let $\beta_{l}(t)=\sin (((l-1 / 2) \pi / T) t)$ on $[0, T]$. Then, $\Theta \equiv\left\{\beta_{l}\right\}_{l=1}^{\infty}$ is an orthogonal sequence in $C_{0}^{\prime}[0, T]$. Additionally, $\widetilde{\Theta}=$ $\left\{(\sqrt{2 T} /(l-1 / 2) \pi) \beta_{l}\right\}_{l=1}^{\infty}$ is a complete orthonormal set in $C_{0}^{\prime}[0, T]$. In fact, $\beta_{l} \in C_{0}^{*}[0, T]$ for all $l \in\{1,2, \ldots\}$. We can take different pair $(\mathscr{H}, \mathscr{G})$ from $\Theta$, which satisfies conditions (c1) and (c2) above. For instance, let $\mathscr{H}=\left\{\beta_{l_{1}}, \ldots, \beta_{l_{n}}\right\}$ and let $\mathscr{G}=\left\{\beta_{k_{1}}, \ldots, \beta_{k_{m}}\right\}$, where $l_{1}<l_{2}<\cdots<l_{n}<k_{1} \leq k_{2} \leq \cdots \leq$ $k_{m}$.
Remark 9. Let $\mathscr{H}=\left\{h_{1}, \ldots, h_{n}\right\}$ and $\mathscr{G}=\left\{g_{1}, \ldots, g_{m}\right\}$ satisfy the conditions (c1) and (c2). Then, the stochastic inner products

$$
\left(h_{j}, A_{s(\mathscr{G})} x\right)^{\sim}=\left(h_{j} \odot s(\mathscr{G}), x\right)^{\sim}, \quad j=1, \ldots, n,
$$

form a set of independent Gaussian random variables on $B$ with mean 0 and variance

$$
\begin{aligned}
\left|h_{j} \odot s(\mathscr{G})\right|^{2} & =\left\langle h_{j} \odot s(\mathscr{G}), h_{j} \odot s(\mathscr{G})\right\rangle \\
& =\left\langle h_{j} \odot s(\mathscr{G}) \odot s(\mathscr{G}), h_{j}\right\rangle \\
& =\left\langle h_{j} \odot\left(\sum_{k=1}^{m} g_{k}^{\odot 2}\right), h_{j}\right\rangle \\
& =\left\langle\sum_{k=1}^{m}\left(h_{j} \odot g_{k}^{\odot 2}\right), h_{j}\right\rangle \\
& =\sum_{k=1}^{m}\left\langle h_{j} \odot g_{k}^{\odot 2}, h_{j}\right\rangle \\
& =\sum_{k=1}^{m}\left\langle h_{j} \odot g_{k}, h_{j} \odot g_{k}\right\rangle \\
& =\sum_{k=1}^{m}\left|h_{j} \odot g_{k}\right|^{2},
\end{aligned}
$$

where $s(\mathscr{G}) \equiv s\left(g_{1}, \ldots, g_{m}\right)$ is given by (33).

Remark 10. Given an orthogonal subset $\mathscr{H}$, let $B_{\mathscr{H}}^{*}$ be the space of every element $g$ in $B^{*}-\{0\}$ such that $\{h \odot g: h \in \mathscr{H}\}$ is orthogonal in $H$. Then, for any finite subset $\mathscr{G}$ of $B_{\mathscr{H}}^{*}$, the pair $(\mathscr{H}, \mathscr{G})$ satisfies conditions (c1) and (c2) above.

Given an orthogonal set $\mathscr{H}$ in $H$, let $\boldsymbol{H}_{\mathscr{H}}^{(1)}$ be the class of all cylinder-type functionals, $F$, given by (9) for $\nu$-a.e. $x \in B$, where the corresponding function $f$ of $F$ satisfies the condition

$$
\int_{\mathbb{R}^{n}}|f(\vec{v})| \exp \left\{-\sum_{j=1}^{n} \frac{v_{j}^{2}}{2\left|h_{j} \odot g\right|^{2}}\right\} d \vec{v}<+\infty
$$

for all $g \in B_{\mathscr{H}}^{*}$.

We are now ready to present our rotation property of abstract Wiener measure associated with admixable operators.

Theorem 11. Let $\mathscr{H}=\left\{h_{1}, \ldots, h_{n}\right\}$ be an orthogonal set in $H$ and let $F \in \mathfrak{G}_{\mathscr{H}}^{(1)}$ be given by (9). Let $\mathscr{G}=\left\{g_{1}, \ldots, g_{m}\right\}$ be a finite subset of $B_{\mathscr{H}}^{*}$, and, for each $k \in\{1, \ldots, m\}$, let $A_{g_{k}}$ be the $g_{k}^{\odot}$-admixable operator on $B$. Then,

$$
\int_{B^{m}} F\left(\sum_{k=1}^{m} A_{g_{k}} x_{k}\right) d \nu^{m}\left(x_{1}, \ldots, x_{m}\right)=\int_{B} F\left(A_{s(\mathscr{G})} x\right) d \nu(x),
$$

where $s(\mathscr{G})$ is given by (33). 
Proof. For each $j \in\{1, \ldots, n\}$ and each $k \in\{1, \ldots, m\}$, let $\alpha_{j}^{k}=h_{j} \odot g_{k}$. For each $k \in\{1, \ldots, m\}, \mathscr{A}_{k}=\left\{\alpha_{1}^{k}, \ldots, \alpha_{n}^{k}\right\}$ is orthogonal in $H$ by condition (c2). Hence, for each $k \in$ $\{1, \ldots, m\}$, the stochastic inner products

$$
\left(h_{j}, A_{g_{k}} x\right)^{\sim}=\left(\alpha_{j}^{k}, x\right)^{\sim}, \quad j=1, \ldots, n,
$$

form a set of independent Gaussian random variables with mean 0 and variance $\left|\alpha_{j}^{k}\right|^{2}$.

We observe that

$$
\begin{aligned}
X_{\mathscr{H}}\left(\sum_{k=1}^{m} A_{g_{k}} x_{k}\right) \\
=\left(\left(h_{1}, \sum_{k=1}^{m} A_{g_{k}} x_{k}\right)^{\sim}, \ldots,\left(h_{n}, \sum_{k=1}^{m} A_{g_{k}} x_{k}\right)^{\sim}\right) \\
=\left(\sum_{k=1}^{m}\left(h_{1}, A_{g_{k}} x_{k}\right)^{\sim}, \ldots, \sum_{k=1}^{m}\left(h_{n}, A_{g_{k}} x_{k}\right)^{\sim}\right) \\
=\sum_{k=1}^{m}\left(\left(h_{1}, A_{g_{k}} x_{k}\right)^{\sim}, \ldots,\left(h_{n}, A_{g_{k}} x_{k}\right)^{\sim}\right) \\
=\sum_{k=1}^{m}\left(\left(\alpha_{1}^{k}, x_{k}\right)^{\sim}, \ldots,\left(\alpha_{n}^{k}, x_{k}\right)^{\sim}\right) \\
=\sum_{k=1}^{m} X_{\mathscr{A}_{k}}\left(x_{k}\right) .
\end{aligned}
$$

Thus, using (9), (39), and (30), we obtain

$$
\begin{aligned}
\int_{B^{m}} F & \left(\sum_{k=1}^{m} A_{g_{k}} x_{k}\right) d \nu^{m}\left(x_{1}, \ldots, x_{m}\right) \\
& =\int_{B^{m}} f\left(X_{\mathscr{H}}\left(\sum_{k=1}^{m} A_{g_{k}} x_{k}\right)\right) d \nu^{m}\left(x_{1}, \ldots, x_{m}\right) \\
& =\int_{B^{m}} f\left(\sum_{k=1}^{m} X_{\mathscr{A}_{k}}\left(x_{k}\right)\right) d \nu^{m}\left(x_{1}, \ldots, x_{m}\right) \\
& =\int_{B} f\left(X_{\mathscr{W}_{(m)}}(x)\right) d \nu(x),
\end{aligned}
$$

where $\mathscr{W}_{(m)}=\left\{w_{1}, \ldots, w_{n}\right\}$ is an orthogonal set in $H$, such that

$$
\left|w_{j}\right|^{2}=\sum_{k=1}^{m}\left|\alpha_{j}^{k}\right|^{2}=\sum_{k=1}^{m}\left|h_{j} \odot g_{k}\right|^{2}
$$

for each $j \in\{1, \ldots, n\}$.
Next, we note that, for each $j, l \in\{1, \ldots, n\}$ with $j \neq l$,

$$
\begin{aligned}
\left\langle h_{j} \odot s(\mathscr{G}), h_{l} \odot s(\mathscr{G})\right\rangle & =\left\langle h_{j} \odot s(\mathscr{G}) \odot s(\mathscr{G}), h_{l}\right\rangle \\
& =\left\langle h_{j} \odot \sum_{k=1}^{m} g_{k}^{\odot 2}, h_{l}\right\rangle \\
& =\left\langle\sum_{k=1}^{m} h_{j} \odot g_{k}^{\odot 2}, h_{l}\right\rangle \\
& =\sum_{k=1}^{m}\left\langle h_{j} \odot g_{k}^{\odot 2}, h_{l}\right\rangle \\
& =\sum_{k=1}^{m}\left\langle h_{j} \odot g_{k}, h_{l} \odot g_{k}\right\rangle=0 .
\end{aligned}
$$

Hence, from (35) and (42), we see that $\left\{h_{1} \odot s(\mathscr{G}), \ldots, h_{n} \odot\right.$ $s(\mathscr{G})\}$ is an orthogonal set in $H$ with $\left|h_{j} \odot s(\mathscr{G})\right|^{2}=\sum_{k=1}^{m} \mid h_{j} \odot$ $\left.g_{k}\right|^{2}$ for all $j \in\{1, \ldots, n\}$, and so we can choose $\mathscr{W}_{(m)}$ to be the orthogonal set $\left\{h_{1} \odot s(\mathscr{G}), \ldots, h_{n} \odot s(\mathscr{G})\right\}$. In this case, we see that

$$
\begin{array}{rl}
\int_{B} & f\left(X_{\mathscr{W}_{(m)}}(x)\right) d \nu(x) \\
\quad & \int_{B} f\left(\left(h_{1} \odot s(\mathscr{G}), x\right)^{\sim}, \ldots,\left(h_{n} \odot s(\mathscr{G}), x\right)^{\sim}\right) d \nu(x) \\
& =\int_{B} f\left(\left(h_{1}, A_{s(\mathscr{G})} x\right)^{\sim}, \ldots,\left(h_{n}, A_{s(\mathscr{G})} x\right)^{\sim}\right) d \nu(x) \\
& =\int_{B} F\left(A_{s(\mathscr{G})} x\right) d v(x) .
\end{array}
$$

Equation (37) follows from (40) and (43).

Example 12. Let us return to the classical Wiener space $\left(C_{0}[0, T], C_{0}^{\prime}[0, T], m_{w}\right)$ again. We introduce the family of functions $\Gamma \equiv\left\{\gamma_{\tau}: \tau \in[0, T]\right\}$ from $C_{0}^{\prime}[0, T]$ :

$$
\gamma_{\tau}(s)= \begin{cases}s, & s \in[0, \tau] \\ \tau, & s \in[\tau, T]\end{cases}
$$

These functions have the reproducing property

$$
\begin{aligned}
\left\langle h, \gamma_{\tau}\right\rangle & =\int_{0}^{T} D h(s) D \gamma_{\tau}(s) d s \\
& =\int_{0}^{T} D h(s) \chi_{[0, \tau]}(s) d s \\
& =\int_{0}^{\tau} D h(s) d s=h(\tau)
\end{aligned}
$$

for all $h \in C_{0}^{\prime}[0, T]$. In fact, $\Gamma \subset C_{0}^{*}[0, T]$. 
For $\gamma_{\tau} \in \Gamma$, we consider the admixable operator $A_{\gamma_{\tau}}$ given by (21). We note that, for each $g \in C_{0}^{*}[0, T]$,

$$
\begin{aligned}
\left(g, A_{\gamma_{\tau}} x\right)^{\sim} & =\left(g \odot \gamma_{\tau}, x\right)^{\sim} \\
& =\int_{0}^{T} D g(s) D \gamma_{\tau}(s) \tilde{d} x(s) \\
& =\int_{0}^{\tau} D g(s) \tilde{d} x(s) \\
& =\mathscr{Z}_{g}(x, \tau)=A_{g} x(\tau) .
\end{aligned}
$$

From this, we see that, for $g_{I}(t)=t=\int_{0}^{t} d s \in C_{0}^{*}[0, T]$ and $a \in \mathbb{R}$,

$$
\left(g_{I}, A_{a \gamma_{\tau}} x\right)^{\sim}=a x(\tau)
$$

and so, using (37), we find that, for any $f \in L_{1}(\mathbb{R})$ and every $a, b \in \mathbb{R}$,

$$
\begin{gathered}
\int_{\left(C_{0}[0, T]\right)^{2}} f\left(a x_{1}(\tau)+b x_{2}(\tau)\right) d\left(m_{w} \times m_{w}\right)\left(x_{1}, x_{2}\right) \\
=\int_{\left(C_{0}[0, T]\right)^{2}} f\left(\left(g_{I}, A_{a \gamma_{\tau}} x_{1}\right)^{\sim}\right. \\
\left.+\left(g_{I}, A_{b \gamma_{\tau}} x_{2}\right)^{\sim}\right) d\left(m_{w} \times m_{w}\right)\left(x_{1}, x_{2}\right) \\
=\int_{C_{0}[0, T]} f\left(\left(g_{I}, A_{s\left(a \gamma_{\tau}, b \gamma_{\tau}\right)} x\right)^{\sim}\right) d m_{w}(x) .
\end{gathered}
$$

Using (16), we now have

$$
\begin{aligned}
s\left(a \gamma_{\tau}, b \gamma_{\tau}\right)(t) & =\sqrt[\odot]{\left(a \gamma_{\tau}\right)^{\odot 2}+\left(b \gamma_{\tau}\right)^{\odot 2}}(t) \\
& =\sqrt{\left(a \gamma_{\tau}\right)^{\odot 2}+\left(b \gamma_{\tau}\right)^{\odot 2}}(t) \\
& =\sqrt{a^{2}+b^{2}} \gamma_{\tau}(t)
\end{aligned}
$$

on $[0, T]$. Thus, by (48), (49), and (47), we have

$$
\begin{gathered}
\int_{\left(C_{0}[0, T]\right)^{2}} f\left(a x_{1}(\tau)+b x_{2}(\tau)\right) d\left(m_{w} \times m_{w}\right)\left(x_{1}, x_{2}\right) \\
=\int_{C_{0}[0, T]} f\left(\sqrt{a^{2}+b^{2}} x(\tau)\right) d m_{w}(x) .
\end{gathered}
$$

Proposition 13 (Cameron and Storvick, [2]). Let $F\left(\sqrt{a^{2}+b^{2}} x\right)$ be Wiener integrable on $C_{0}[0, T]$ for $a, b \in \mathbb{R}$. As a result, $F\left(a x_{1}+b x_{2}\right)$ is integrable on $C_{0}^{2}[0, T]$, and

$$
\begin{gathered}
\int_{C_{0}^{2}[0, T]} F\left(a x_{1}+b x_{2}\right) d\left(m_{w} \times m_{w}\right)\left(x_{1}, x_{2}\right) \\
=\int_{C_{0}[0, T]} F\left(\sqrt{a^{2}+b^{2}} x\right) d m_{w}(x) .
\end{gathered}
$$

Proof. Let $n$ be any positive integer, and let $0=\tau_{0}<\tau_{1}<$ $\cdots<\tau_{n} \leq T$ be any partition of $[0, T]$. It suffices to show that (51) holds for any tame function

$$
F(x)=f\left(x\left(\tau_{1}\right), x\left(\tau_{2}\right)-x\left(\tau_{1}\right), \ldots, x\left(\tau_{n}\right)-x\left(\tau_{n-1}\right)\right)
$$

with $f \in L_{1}\left(\mathbb{R}^{n}\right)$.

Let $\odot$ be defined by (16) between $C_{0}^{\prime}[0, T]$ and $C_{0}^{*}[0, T]$. For $g_{I}(t)=t=\int_{0}^{t} d s \in C_{0}^{*}[0, T]$, let the $g_{I}^{\odot}$-admixable operator $A_{g_{I}}$ be given by (21), and, for each $j \in\{1, \ldots, n\}$, let

$$
h_{j}(t)=\int_{0}^{t} \chi_{\left[\tau_{j-1}, \tau_{j}\right]}(s) d s
$$

on $[0, T]$. For any $a, b \in \mathbb{R}-\{0\}$,

(c1) $\left\{h_{1}, \ldots, h_{n}\right\}$ is an orthogonal set in $C_{0}^{\prime}[0, T]$, and

(c2) $\left\{h_{1} \odot a g_{I}, \ldots, h_{n} \odot a g_{I}\right\}$ and $\left\{h_{1} \odot b g_{I}, \ldots, h_{n} \odot b g_{I}\right\}$ are orthogonal sets in $C_{0}^{\prime}[0, T]$.

Also, for any $a, b \in \mathbb{R}-\{0\}, A_{s\left(a g_{I}, b g_{I}\right)}=A_{\sqrt{a^{2}+b^{2}} g_{I}}$, and, for each $j \in\{1, \ldots, n\}$,

$$
\begin{aligned}
\left(h_{j}, A_{a g_{I}} x\right)^{\sim} & =a \int_{0}^{T} \chi_{\left[\tau_{j-1}, \tau_{j}\right]}(s) d x(s) \\
& =a\left(x\left(\tau_{j}\right)-x\left(\tau_{j-1}\right)\right) .
\end{aligned}
$$

Thus, the left side of (51) with $F$ given by (52) is rewritten by

$$
\begin{gathered}
\int_{\left(C_{0}[0, T]\right)^{2}} F\left(a x_{1}+b x_{2}\right) d\left(m_{w} \times m_{w}\right)\left(x_{1}, x_{2}\right) \\
=\int_{\left(C_{0}[0, T]\right)^{2}} f\left(\left(h_{1}, A_{a g_{I}} x_{1}\right)^{\sim}\right. \\
+\left(h_{1}, A_{b g_{I}} x_{2}\right)^{\sim} \\
\ldots,\left(h_{n}, A_{a g_{I}} x_{1}\right)^{\sim} \\
\left.+\left(h_{n}, A_{b g_{I}} x_{2}\right)^{\sim}\right) d\left(m_{w} \times m_{w}\right)\left(x_{1}, x_{2}\right) .
\end{gathered}
$$

Thus, from Theorem 11, we obtain

$$
\begin{gathered}
\int_{\left(C_{0}[0, T]\right)^{2}} F\left(a x_{1}+b x_{2}\right) d\left(m_{w} \times m_{w}\right)\left(x_{1}, x_{2}\right) \\
=\int_{C_{0}[0, T]} f\left(\left(h_{1}, A_{\sqrt{a^{2}+b^{2}} g_{I}} x\right)^{\sim}\right. \\
\left.\ldots,\left(h_{n}, A_{\sqrt{a^{2}+b^{2}} g_{I}} x\right)^{\sim}\right) d m_{w}(x) \\
=\int_{C_{0}[0, T]} F\left(\sqrt{a^{2}+b^{2}} x\right) d m_{w}(x) .
\end{gathered}
$$

Using standard methods, similar to those in [1], we can obtain the result for general functionals $F$ on $C_{0}[0, T]$. 


\section{Fourier-Feynman Transforms and Convolutions Associated with Admixable Operators}

In this section, to apply our results from the previous section, we first define an $L_{p}$ analytic Fourier-Feynman transform associated with admixable operators on $B$. Then, we establish the existence theorem and the inverse transform theorem of this transform for some classes of cylinder-type functionals on $B$ having the form (9) for $s$-a.e. $x \in B$. Moreover, we present various relationships involving the convolution and the transforms.

Throughout the rest of this paper, let $\mathbb{C}, \mathbb{C}_{+}$, and $\widetilde{\mathbb{C}}_{+}$ denote, respectively, the complex numbers, the complex numbers with positive real part, and the nonzero complex numbers with nonnegative real part.

Let $g \in B^{*}$, and let $A_{g}$ be the corresponding admixable operator on $B$. Let $F: B \rightarrow \mathbb{C}$ be a scale-invariant measurable functional such that

$$
J_{F}(g ; \lambda)=\int_{B} F\left(\lambda^{-1 / 2} A_{g} x\right) d \nu(x)
$$

exists as a finite number for all $\lambda>0$. If there exists a function $J_{F}^{*}(g ; \lambda)$ analytic on $\mathbb{C}_{+}$such that $J_{F}^{*}(g ; \lambda)=J_{F}(g ; \lambda)$ for all $\lambda>0$, then $J_{F}^{*}(g ; \lambda)$ is defined to be the analytic Wiener integral (associated with the $g^{\odot}$-admixable operator $A_{g}$ ) of $F$ over $B$ with parameter $\lambda$. For $\lambda \in \mathbb{C}_{+}$, we write

$$
I_{g}^{\mathrm{anw}_{\lambda}}[F]=J_{F}^{*}(g ; \lambda)
$$

Let $q \neq 0$ be a real number, and let $F$ be a functional such that $\int_{B}^{\mathrm{anw}_{\lambda}} F\left(A_{g} x\right) d \nu(x)=J_{F}^{*}(g ; \lambda)$ exists for all $\lambda \in \mathbb{C}_{+}$. If the following limit exists, we call it the analytic Feynman integral of $F$ with parameter $q$, and we write

$$
I_{g}^{\operatorname{anf}_{q}}[F]=\lim _{\lambda \rightarrow-i q} I_{g}^{\mathrm{anw}_{\lambda}}[F]
$$

where $\lambda$ approaches -iq through values in $\mathbb{C}_{+}$.

Note that if $A_{g}$ is the identity operator on $B$, then these definitions agree with the previous definitions of the analytic Wiener integral and the analytic Feynman integral [18-20].

We are now ready to state the definition of the analytic Fourier-Feynman transform associated with admixable operator (admix-FFT).

Definition 14. Let $(B, H, v)$ be an abstract Wiener space. For $g \in B^{*}, \lambda \in \mathbb{C}_{+}$, and $y \in B$, let

$$
T_{\lambda, g}(F)(y)=\int_{B}^{\mathrm{anw}_{\lambda}} F\left(y+A_{g} x\right) d \nu(x),
$$

where $A_{g}$ is the $g^{\odot}$-admixable operator on $B$. Let $q$ be a nonzero real number. For $p \in(1,2]$, we define the $L_{p}$ analytic $g^{\odot}$-admix-FFT, $T_{q, g}^{(p)}(F)$ of $F$, by the formula $\left(\lambda \in \mathbb{C}_{+}\right)$,

$$
T_{q, g}^{(p)}(F)(y)=\underset{\lambda \rightarrow-i q}{\lim . T_{\lambda, g}}(F)(y),
$$

if it exists; that is, for each $\rho>0$,

$$
\lim _{\lambda \rightarrow-i q} \int_{B}\left|T_{\lambda, g}(F)(\rho y)-T_{q, g}^{(p)}(F)(\rho y)\right|^{p^{\prime}} d \nu(y)=0,
$$

where $1 / p+1 / p^{\prime}=1$. We define the $L_{1}$ analytic $g^{\odot}$-admixFFT, $T_{q, g}^{(1)}(F)$ of $F$, by the formula $\left(\lambda \in \mathbb{C}_{+}\right)$,

$$
T_{q, g}^{(1)}(F)(y)=\lim _{\lambda \rightarrow-i q} T_{\lambda, g}(F)(y),
$$

if it exists.

We note that, for $p \in[1,2], T_{q, g}^{(p)}(F)$ is defined only $s$-a.e.. We also note that if $T_{q, g}^{(p)}(F)$ exists and if $F \approx G$, then $T_{q, g}^{(p)}(G)$ exists and $T_{q, g}^{(p)}(G) \approx T_{q, g}^{(p)}(F)$. (CP).

Next, we give the definition of the convolution product

Definition 15. Let $F$ and $G$ be scale-invariant measurable functionals on $B$. For $\lambda \in \widetilde{\mathbb{C}}_{+}$and $g \in B^{*}$, we define their $\mathrm{CP}$ with respect to $A_{g}$ (if it exists) by

$$
\begin{aligned}
& (F * G)_{\lambda, g}(y) \\
& \quad=\left\{\begin{aligned}
& \int_{B}^{\text {anw }} F\left(\frac{y+A_{g} x}{\sqrt{2}}\right) G\left(\frac{y-A_{g} x}{\sqrt{2}}\right) d \nu(x), \\
& \lambda \in \mathbb{C}_{+}, \\
& \int_{B}^{\text {anf }_{q}} F\left(\frac{y+A_{g} x}{\sqrt{2}}\right) G\left(\frac{y-A_{g} x}{\sqrt{2}}\right) d \nu(x), \\
& \lambda=-i q, q \in \mathbb{R}, q \neq 0 .
\end{aligned}\right.
\end{aligned}
$$

When $\lambda=-i q$, we denote $(F * G)_{\lambda, g}$ by $(F * G)_{q, g}$.

For any scale-invariant measurable functional $F$, we see that, for $\lambda>0$,

$$
\int_{B}^{\mathrm{anw} \lambda} F\left(y+A_{g} x\right) d \nu(x)=\int_{B} F\left(y+\lambda^{-1 / 2} A_{g} x\right) d \nu(x)
$$

if it exists.

Let $\mathscr{M}\left(\mathbb{R}^{n}\right)$ denote the space of complex-valued, countably additive (and hence finite) Borel measures on $\mathscr{B}\left(\mathbb{R}^{n}\right)$, the Borel $\sigma$-algebra of $\mathbb{R}^{n}$. It is well known that a complexvalued Borel measure $\tau$ necessarily has a finite total variation $\|\tau\|$, and $\mathscr{M}\left(\mathbb{R}^{n}\right)$ is a Banach algebra under the norm $\|\cdot\|$ and with convolution as multiplication.

For $\tau \in \mathscr{M}\left(\mathbb{R}^{n}\right)$, the Fourier transform $\widehat{\tau}$ of $\tau$ is a complexvalued function defined on $\mathbb{R}^{n}$ by the formula

$$
\widehat{\tau}(\vec{u})=\int_{\mathbb{R}^{n}} \exp \left\{i \sum_{j=1}^{n} u_{j} v_{j}\right\} d \tau(\vec{v})
$$

where $\vec{u}=\left(u_{1}, \ldots, u_{n}\right)$ and $\vec{v}=\left(v_{1}, \ldots, v_{n}\right)$ are in $\mathbb{R}^{n}$.

Let $\mathscr{H}=\left\{h_{1}, \ldots, h_{n}\right\}$ be an orthonormal set in $H$. Define the functional $F: B \rightarrow \mathbb{C}$ by

$$
F(x)=\widehat{\tau}\left(X_{\mathscr{H}}(x)\right)=\widehat{\tau}\left(\left(h_{1}, x\right)^{\sim}, \ldots,\left(h_{n}, x\right)^{\sim}\right)
$$


for $s$-a.e. $x \in B$, where $\widehat{\tau}$ is the Fourier transform of $\tau$ in $\mathscr{M}\left(\mathbb{R}^{n}\right)$. Then $F$ is a bounded cylinder-type functional because $|\widehat{\tau}(\vec{u})| \leq\|\tau\|<+\infty$.

Let $\mathfrak{I}_{\mathscr{H}}$ be the set of all functionals $F$ on $B$ having the form (67). Note that $F \in \mathfrak{I}_{\mathscr{H}}$ implies that $F$ is scaleinvariant measurable on $B$. Throughout this section, we fix the orthogonal set $\mathscr{H}$.

We now state the existence theorem for the analytic Feynman integral of the functionals in $\mathfrak{I}_{\mathscr{H}}$.

Theorem 16. Let $F \in \mathfrak{T}_{\mathscr{H}}$ be given by (67). Then, for all $g \in B_{\mathscr{H}}^{*}$ and all nonzero real numbers $q$, the analytic Feynman integral $I_{g}^{a n f} f_{a}[F]$ of $F$ exists and is given by the formula

$$
I_{g}^{a n f_{q}}[F]=\int_{\mathbb{R}^{n}} \exp \left\{-\frac{i}{2 q} \sum_{j=1}^{n}\left|h_{j} \odot g\right|^{2} v_{j}^{2}\right\} d \tau(\vec{v}) .
$$

Proof. By (67), (66), the Fubini theorem, (23), and (24), we see that, for all $\lambda>0$,

$$
\begin{aligned}
J_{F} & (g ; \lambda) \\
= & \int_{B} F\left(\lambda^{-1 / 2} A_{g} x\right) d \nu(x) \\
= & \int_{\mathbb{R}^{n}}\left[\int_{B} \exp \left\{i \lambda^{-1 / 2} \sum_{j=1}^{n}\left(h_{j} \odot g, x\right)^{\sim} v_{j}\right\} d \nu(x)\right] d \tau(\vec{v}) \\
= & \left(\prod_{j=1}^{n} \frac{\lambda}{2 \pi\left|h_{j} \odot g\right|^{2}}\right)^{1 / 2} \\
& \times \int_{\mathbb{R}^{n}}\left[\int_{\mathbb{R}^{n}} \exp \left\{i \sum_{j=1}^{n} u_{j} v_{j}-\frac{\lambda}{2} \sum_{j=1}^{n} \frac{u_{j}^{2}}{\left|h_{j} \odot g\right|^{2}}\right\} d \vec{u}\right] d \tau(\vec{v}) \\
= & \left(\prod_{j=1}^{n} \frac{\lambda}{2 \pi\left|h_{j} \odot g\right|^{2}}\right)^{1 / 2} \int_{\mathbb{R}^{n}}\left[\prod_{j=1}^{n} \int_{\mathbb{R}} \exp \left\{i u_{j} v_{j}-\frac{\lambda}{2} \frac{u_{j}^{2}}{\left|h_{j} \odot g\right|^{2}}\right\} d u_{j}\right] d \tau(\vec{v}) \\
& \times \int_{\mathbb{R}^{n}} \exp \left\{-\frac{1}{2 \lambda} \sum_{j=1}^{n}\left|h_{j} \odot g\right|^{2} v_{j}^{2}\right\} d \tau(\vec{v}) .
\end{aligned}
$$

Now, let $J_{F}^{*}(g ; \lambda)=\int_{\mathbb{R}^{n}} \exp \left\{-(1 / 2 \lambda) \sum_{j=1}^{n}\left|h_{j} \odot g\right|^{2} v_{j}^{2}\right\} d \tau(\vec{v})$ for $\lambda \in \mathbb{C}_{+}$. Then, $J_{F}^{*}(g ; \lambda)=J_{F}(g ; \lambda)$ for all $\lambda>0$ and $\left|J_{F}^{*}(g ; \lambda)\right| \leq \int_{\mathbb{R}^{n}} d|\tau|(\vec{v}) \leq\|\tau\|<\infty$ for all $\lambda \in \mathbb{C}_{+}$. Thus, applying the dominated convergence theorem, we see that $J_{F}^{*}(g ; \lambda)$ is continuous on $\mathbb{C}_{+}$. Also, because $k(\lambda) \equiv$ $\exp \left\{-(1 / 2 \lambda) \sum_{j=1}^{n}\left|h_{j} \odot g\right|^{2} v_{j}^{2}\right\}$ is analytic on $\mathbb{C}_{+}$, applying the Fubini theorem, we have

$$
\int_{\Delta} J_{F}^{*}(g ; \lambda) d \lambda=\int_{\mathbb{R}^{n}} \int_{\Delta} k(\lambda) d \lambda d \nu(\vec{v})=0
$$

for all rectifiable simple closed curve $\Delta$ lying in $\mathbb{C}_{+}$. Thus, by the Morera theorem, $J_{F}^{*}(g ; \lambda)$ is analytic on $\mathbb{C}_{+}$. Therefore, the analytic Wiener integral $I_{g}^{\text {anw }_{\lambda}}[F]=J_{F}^{*}(g ; \lambda)$ exists. Finally, applying the dominated convergence theorem, we know that $I_{g}^{\text {anf }_{q}}[F]=\lim _{\lambda \rightarrow-i q} I_{g}^{\text {anw }}{ }_{\lambda}[F]$ is given by the right side of (68).

Next, we establish the existence of the admix-FFT for functionals in $\mathfrak{I}_{\mathscr{H}}$.

Theorem 17. Let $F \in \mathfrak{I}_{\mathscr{H}}$ be given by (67). Then, for all $p \in$ $[1,2]$ and all $g \in B^{*}$, the analytic $L_{p} g^{\odot}$-admix-FFT, $T_{q, g}^{(p)}(F)$, exists for all nonzero real numbers $q$, belongs to $\mathfrak{I}_{\mathscr{H}}$, and is given by the formula

$$
T_{q, g}^{(p)}(F)(y)=\int_{\mathbb{R}^{n}} \exp \left\{i \sum_{j=1}^{n}\left(h_{j}, y\right)^{\sim} v_{j}\right\} d \tau_{g}^{q}(\vec{v})
$$

for s-a.e. $y \in B$, where $\tau_{g}^{q}$ is the complex measure on $\mathbb{R}^{n}$ given by

$$
\tau_{g}^{q}(E)=\int_{E} \exp \left\{-\frac{i}{2 q} \sum_{j=1}^{n}\left|h_{j} \odot g\right|^{2} v_{j}^{2}\right\} d \tau(\vec{v})
$$

for $E \in \mathscr{B}\left(\mathbb{R}^{n}\right)$.

Proof. Proceeding as in the proof of Theorem 16, we see that, for all $\lambda \in \mathbb{C}_{+}$, all $g \in B_{\mathscr{H}}^{*}$, and for $s$-a.e. $y \in B$,

$$
\begin{aligned}
& T_{\lambda, g}(F)(y) \\
& \quad=\int_{B} F\left(y+\lambda^{-1 / 2} A_{g} x\right) d v(x) \\
& \quad=\int_{\mathbb{R}^{n}} \exp \left\{i \sum_{j=1}^{n}\left(h_{j}, y\right)^{\sim} v_{j}-\frac{1}{2 \lambda} \sum_{j=1}^{n}\left|h_{j} \odot g\right|^{2} v_{j}^{2}\right\} d \tau(\vec{v}),
\end{aligned}
$$

is an analytic function of $\lambda$ on $\mathbb{C}_{+}$, and that for any $q \in \mathbb{R}-\{0\}$ and $s$-a.e. $y \in B$,

$$
\begin{aligned}
T_{q, g}^{(1)} & (F)(y) \\
& =\lim _{\lambda \rightarrow-i q} T_{\lambda, g}(F)(y) \\
& =\int_{\mathbb{R}^{n}} \exp \left\{i \sum_{j=1}^{n}\left(h_{j}, y\right)^{\sim} v_{j}-\frac{i}{2 q} \sum_{j=1}^{n}\left|h_{j} \odot g\right|^{2} v_{j}^{2}\right\} d \tau(\vec{v}) .
\end{aligned}
$$

Clearly, the set function $\tau_{g}^{q}$ given by (72) is a complex measure on $\mathscr{B}\left(\mathbb{R}^{n}\right)$, and so the right side of (74) can be rewritten as the right side of (71).

Next, we note that

$$
\left|T_{\lambda, g}(F)(y)\right| \leq \int_{\mathbb{R}^{n}} d|\tau|(\vec{v})=\|\tau\|
$$


for all $\lambda \in \mathbb{C}_{+}$and

$$
\left|T_{q, g}^{(1)}(F)(y)\right| \leq \int_{\mathbb{R}^{n}} d|\tau|(\vec{v})=\|\tau\|
$$

Using these, we see that, for all $p \in(1,2]$, all $\rho>0$, and all $\lambda \in \mathbb{C}_{+}$,

$$
\left|T_{\lambda, g}(F)(\rho y)-T_{q, g}^{(1)}(F)(\rho y)\right|^{p^{\prime}} \leq(2\|\tau\|)^{p^{\prime}},
$$

and so, by the dominated convergence theorem, we see that, for any nonzero real $q$,

$$
\lim _{\lambda \rightarrow-i q} \int_{B}\left|T_{\lambda, g}(F)(\rho y)-T_{q, g}^{(1)}(F)(\rho y)\right|^{p^{\prime}} d \nu(y)=0 .
$$

Hence, $T_{q, g}^{(p)}(F)(y)$ exists and is given by the right side of (74) for all desired values of $p$ and $q$ and all $g \in B_{\mathscr{H}}^{*}$. Thus, the theorem is proved.

Theorem 18. Let $F \in \mathfrak{I}_{\mathscr{H}}$ be given by (67). Then, for all $p \in$ $[1,2]$, all $g \in B_{\mathscr{H}}^{*}$, and all nonzero real $q$,

$$
T_{-q, g}^{(p)}\left(T_{q, g}^{(p)}(F)\right) \approx F
$$

As such, the admix-FFT, $T_{q, g}^{(p)}$, has the inverse transform $\left\{T_{q, g}^{(p)}\right\}^{-1}=T_{-q, g}^{(p)}$.

Theorem 19. Let $F$ and $G$ be elements of $\mathfrak{I}_{\mathscr{H}}$ with corresponding finite Borel measures $\tau$ and $\mu$ in $\mathscr{M}(H)$. Then, for all $k \in$ $B_{\mathscr{H}}^{*}$, the $C P,(F * G)_{q, k}$, exists for all nonzero real numbers $q$, belongs to $\mathfrak{I}_{\mathscr{H}}$, and is given by the formula

$$
\begin{aligned}
& (F * G)_{q, k}(y) \\
& \quad=\iint_{\mathbb{R}^{n}} \exp \left\{i \sum_{j=1}^{n}\left(h_{j}, y\right)^{\sim} r_{j}\right\} d\left(\omega_{k}^{q} \circ \varphi^{-1}\right)(h)
\end{aligned}
$$

for s-a.e. $y \in B$, where $\varphi: \mathbb{R}^{n} \times \mathbb{R}^{n} \rightarrow \mathbb{R}^{n}$ is a continuous function defined by

$$
\varphi(\vec{v}, \vec{w})=\frac{(\vec{v}+\vec{w})}{\sqrt{2}}
$$

and $\omega_{k}^{q}$ is a complex measure on $\mathscr{B}\left(\mathbb{R}^{n}\right)$ given by

$$
\begin{aligned}
& \omega_{k}^{q}(E) \\
& \quad=\int_{E} \exp \left\{-\frac{i}{4 q} \sum_{j=1}^{n}\left|h_{j} \odot k\right|^{2}\left(v_{j}-w_{j}\right)^{2}\right\} d \tau(\vec{v}) d \mu(\vec{w})
\end{aligned}
$$

for $E \in \mathscr{B}\left(\mathbb{R}^{n}\right)$.
Proof. Using (64), the Fubini theorem, (23), and (24), we have that, for all $\lambda>0$ and $s$-a.e. $y \in B$,

$$
\begin{aligned}
& (F * G)_{\lambda, k}(y) \\
& =\int_{B} F\left(\frac{y+A_{k} x}{\sqrt{2}}\right) G\left(\frac{y-A_{k} x}{\sqrt{2}}\right) d \nu(x) \\
& =\iint_{\mathbb{R}^{n}} \exp \left\{\frac{i}{\sqrt{2}} \sum_{j=1}^{n}\left(h_{j}, y\right)^{\sim}\left(v_{j}+w_{j}\right)\right\} \\
& \times\left[\int _ { B } \operatorname { e x p } \left\{\frac{i}{\sqrt{2 \lambda}} \sum_{j=1}^{n}\left(h_{j} \odot k, x\right)^{\sim}\right.\right. \\
& =\iint_{\mathbb{R}^{n}} \exp \left\{\frac{i}{\sqrt{2}} \sum_{j=1}^{n}\left(h_{j}, y\right)^{\sim}\left(v_{j}+w_{j}\right)\right. \\
& \left.-\frac{1}{4 \lambda} \sum_{j=1}^{n}\left|h_{j} \odot k\right|^{2}\left(v_{j}-w_{j}\right)^{2}\right\} d \tau(\vec{v}) d \mu(\vec{w}) .
\end{aligned}
$$

Using the same argument as in the proof of Theorem 17, we can show that the last expression in the previous equation is an analytic function of $\lambda$ on $\mathbb{C}_{+}$and is a bounded continuous function of $\lambda$ on $\widetilde{\mathbb{C}}_{+}$because $\tau$ and $\mu$ are finite Borel measures. Hence, $(F * G)_{q, k}$ exists and is given by

$$
\begin{aligned}
&(F * G)_{q, k}(y) \\
&=\iint_{\mathbb{R}^{\mathrm{n}}} \exp \left\{\frac{i}{\sqrt{2}} \sum_{j=1}^{n}\left(h_{j}, y\right)^{\sim}\left(v_{j}+w_{j}\right)\right. \\
&\left.\quad-\frac{i}{4 q} \sum_{j=1}^{n}\left|h_{j} \odot k\right|^{2}\left(v_{j}-w_{j}\right)^{2}\right\} d \tau(\vec{v}) d \mu(\vec{w})
\end{aligned}
$$

for all $q \in \mathbb{R}-\{0\}$ and $s$-a.e. $y \in B$.

Consider the set function $\omega_{k}^{q}$ and the continuous function $\varphi$ given by (82) and (81), respectively. Clearly, the set function $\omega_{k}^{q}$ is a complex measure on $\mathscr{B}\left(\mathbb{R}^{n}\right)$. Hence, $\omega_{k}^{q} \circ \varphi$ is an element of $\mathscr{M}\left(\mathbb{R}^{n}\right)$, and so the right side of (84) can be rewritten as the right side of (80). Thus, the theorem is proved.

Lemma 20. Let $\mathscr{H}=\left\{h_{1}, \ldots, h_{n}\right\}$ be any orthogonal set in $H$. For every $g \in B_{\mathscr{H}}^{*}$, every $\vec{v}=\left(v_{1}, \ldots, v_{n}\right)$ and $\vec{w}=\left(w_{1}, \ldots, w_{n}\right)$ in $\mathbb{R}^{n}$, let $Y_{g, \vec{v}}^{\mathscr{H}}, Z_{g, \vec{w}}^{\mathscr{H}}: B^{2} \rightarrow \mathbb{R}$ be given by

$$
\begin{aligned}
& Y_{g, \vec{v}}^{\mathscr{H}}\left(x_{1}, x_{2}\right)=\sum_{j=1}^{n} \frac{\left(h_{j}, A_{g} x_{1}+A_{g} x_{2}\right)^{\sim}}{\sqrt{2}} v_{j}, \\
& Z_{g, \vec{w}}^{\mathscr{H}}\left(x_{1}, x_{2}\right)=\sum_{j=1}^{n} \frac{\left(h_{j}, A_{g} x_{1}-A_{g} x_{2}\right)^{\sim}}{\sqrt{2}} w_{j},
\end{aligned}
$$


respectively. As a result, $Y_{g, \vec{v}}^{\mathscr{H}}$ and $Z_{g, \vec{w}}^{\mathscr{H}}$ are independent random variables.

Proof. Since the random variables $Y_{g, \vec{v}}^{\mathscr{H}}$ and $Z_{g, \vec{w}}^{\mathscr{H}}$ are Gaussian with mean zero, it suffices to show that

$$
\int_{B^{2}} Y_{g, \vec{v}}^{\mathscr{H}}\left(x_{1}, x_{2}\right) Z_{g, \vec{w}}^{\mathscr{H}}\left(x_{1}, x_{2}\right) d(\nu \times v)\left(x_{1}, x_{2}\right)=0 .
$$

We know that $\left\{h_{j} \odot g, \ldots, h_{n} \odot g\right\}$ is an orthogonal set in $H$; thus, $\left\{\left(h_{j} \odot g, x\right)^{\sim}, \ldots,\left(h_{n} \odot g, x\right)^{\sim}\right\}$ is a set of independent Gaussian random variables with mean zero on $B$. However, using the Fubini theorem, we obtain

$$
\begin{aligned}
& \int_{B^{2}} Y_{g, \vec{v}}^{\mathscr{H}}\left(x_{1}, x_{2}\right) Z_{g, \vec{w}}^{\mathscr{H}}\left(x_{1}, x_{2}\right) d(\nu \times \nu)\left(x_{1}, x_{2}\right) \\
& =\frac{1}{2} \sum_{j=1}^{n} \sum_{k=1}^{n}\left\{v_{j} w_{k} \iint_{B}\left(h_{j} \odot g, x_{1}\right)^{\sim}\right. \\
& \times\left(h_{k} \odot g, x_{1}\right)^{\sim} d \nu\left(x_{1}\right) d \nu\left(x_{2}\right) \\
& +v_{j} w_{k} \int_{B}\left(h_{j} \odot g, x_{1}\right)^{\sim} d v\left(x_{1}\right) \\
& \times \int_{B}\left(h_{k} \odot g, x_{2}\right)^{\sim} d v\left(x_{2}\right) \\
& +v_{j} w_{k} \int_{B}\left(h_{j} \odot g, x_{2}\right)^{\sim} d \nu\left(x_{2}\right) \\
& \times \int_{B}\left(h_{k} \odot g, x_{1}\right)^{\sim} d \nu\left(x_{1}\right) \\
& -v_{j} w_{k} \iint_{B}\left(h_{j} \odot g, x_{2}\right)^{\sim} \\
& \left.\times\left(h \odot g, x_{2}\right)^{\sim} d \nu\left(x_{1}\right) d v\left(x_{2}\right)\right\} \\
& =\frac{1}{2} \sum_{j=1}^{n} \sum_{k=1}^{n}\left\{v_{j} w_{k} \int_{B}\left(h_{j} \odot g, x_{1}\right)^{\sim}\left(h_{k} \odot g, x_{1}\right)^{\sim} d \nu\left(x_{1}\right)\right. \\
& -v_{j} w_{k} \int_{B}\left(h_{j} \odot g, x_{2}\right)^{\sim} \\
& \left.\times\left(h_{k} \odot g, x_{2}\right)^{\sim} d \nu\left(x_{2}\right)\right\} \\
& =\frac{1}{2} \sum_{j=1}^{n}\left\{v_{j} w_{j} \int_{B}\left[\left(h_{j} \odot g, x_{1}\right)^{\sim}\right]^{2} d \nu\left(x_{1}\right)\right. \\
& \left.-v_{j} w_{j} \int_{B}\left[\left(h_{j} \odot g, x_{2}\right)^{\sim}\right] d v\left(x_{2}\right)\right\} \\
& +\frac{1}{2} \sum_{j \neq k}^{n}\left\{v_{j} w_{k} \int_{B}\left(h_{j} \odot g, x_{1}\right)^{\sim}\left(h_{k} \odot g, x_{1}\right)^{\sim} d \nu\left(x_{1}\right)\right. \\
& \left.-v_{j} w_{k} \int_{B}\left(h_{j} \odot g, x_{2}\right)^{\sim}\left(h \odot g, x_{2}\right)^{\sim} d \nu\left(x_{2}\right)\right\}
\end{aligned}
$$$$
=0 \text {, }
$$

which concludes the proof of Lemma 20.
Remark 21. For each $\vec{v} \in \mathbb{R}^{n}$, let

$$
\psi(\vec{v} ; x)=\exp \left\{i \sum_{j=1}^{n}\left(h_{j}, x\right)^{\sim} v_{j}\right\}, \quad x \in B .
$$

As such, (67) is rewritten as

$$
F(x)=\int_{\mathbb{R}^{n}} \psi(\vec{v} ; x) d \tau(\vec{v}),
$$

and the functional $\psi\left(\vec{v}_{;} \cdot\right)$ is an element of $\boldsymbol{G}_{\mathscr{H}}^{(1)}$ for each $\vec{v}$.

Applying the same method as used in the proof of Theorem 3.5 in [4], we have the following theorem. For the proof of Theorem 22, we can apply Lemma 20 and Theorem 11 to the functional $\psi$ given by (88).

Theorem 22. Let F, G, $\tau$, and $\mu$ be as in Theorem 19, and let $g$ be an element of $B_{\mathscr{H}}^{*}$. Then, for all $p \in[1,2]$ and all nonzero real $q$,

$$
T_{q, g}^{(p)}\left((F * G)_{q, g}\right)(y)=T_{q, g}^{(p)}(F)\left(\frac{y}{\sqrt{2}}\right) T_{q, g}^{(p)}(G)\left(\frac{y}{\sqrt{2}}\right)
$$

for s-a.e. $y \in B$.

\section{Acknowledgments}

The authors would like to express their gratitude to the referees for their valuable comments and suggestions which have improved the original paper. This research was supported by Basic Science Research Program through the National Research Foundation of Korea (NRF) funded by the Ministry of Education, Science and Technology (2011-0014552).

\section{References}

[1] J. E. Bearman, "Rotations in the product of two Wiener spaces," Proceedings of the American Mathematical Society, vol. 3, pp. 129-137, 1952.

[2] R. H. Cameron and D. A. Storvick, "An operator valued YehWiener integral, and a Wiener integral equation," Indiana University Mathematics Journal, vol. 25, no. 3, pp. 235-258, 1976.

[3] G. W. Johnson and D. L. Skoug, "Scale-invariant measurability in Wiener space," Pacific Journal of Mathematics, vol. 83, no. 1, pp. 157-176, 1979.

[4] J. G. Choi, D. Skoug, and S. J. Chang, "A multiple generalized Fourier-Feynman transform via a rotation on Wiener space," International Journal of Mathematics, vol. 23, no. 7, Article ID 1250068, 20 pages, 2012.

[5] J. G. Choi and S. J. Chang, "A rotation on Wiener space with applications," ISRN Applied Mathematics, vol. 2012, Article ID 578174, 13 pages, 2012.

[6] L. Gross, "Abstract Wiener spaces," in Proceedings of the 5th Berkeley Symposium on Mathematical Statistics and Probability, vol. 2, pp. 31-42, 1965.

[7] D. M. Chung, "Scale-invariant measurability in abstract Wiener spaces," Pacific Journal of Mathematics, vol. 130, no. 1, pp. 27-40, 1987. 
[8] G. Kallianpur and C. Bromley, "Generalized Feynman integrals using analytic continuation in several complex variables," in Stochastic Analysis and Applications, M. Pinsky, Ed., vol. 7, pp. 217-267, Marcel Dekker, New York, NY, USA, 1984.

[9] H. H. Kuo, Gaussian Measures in Banach Spaces, vol. 463 of Lecture Notes in Mathematics, Springer, Berlin, Germany, 1975.

[10] G. W. Johnson and D. L. Skoug, "Notes on the Feynman integral. II," Journal of Functional Analysis, vol. 41, no. 3, pp. 277-289, 1981.

[11] R. E. A. C. Paley, N. Wiener, and A. Zygmund, "Notes on random functions," Mathematische Zeitschrift, vol. 37, no. 1, pp. 647-668, 1933.

[12] C. Park and D. Skoug, "A note on Paley-Wiener-Zygmund stochastic integrals," Proceedings of the American Mathematical Society, vol. 103, no. 2, pp. 591-601, 1988.

[13] C. Park and D. Skoug, "A Kac-Feynman integral equation for conditional Wiener integrals," Journal of Integral Equations and Applications, vol. 3, no. 3, pp. 411-427, 1991.

[14] D. M. Chung, C. Park, and D. Skoug, "Generalized Feynman integrals via conditional Feynman integrals," The Michigan Mathematical Journal, vol. 40, no. 2, pp. 377-391, 1993.

[15] T. Huffman, C. Park, and D. Skoug, "Generalized transforms and convolutions," International Journal of Mathematics and Mathematical Sciences, vol. 20, no. 1, pp. 19-32, 1997.

[16] C. Park and D. Skoug, "Generalized Feynman integrals: the $L\left(L_{2}, L_{2}\right)$ theory," The Rocky Mountain Journal of Mathematics, vol. 25, no. 2, pp. 739-756, 1995.

[17] C. Park and D. Skoug, "Conditional Fourier-Feynman transforms and conditional convolution products," Journal of the Korean Mathematical Society, vol. 38, no. 1, pp. 61-76, 2001.

[18] J. M. Ahn, K. S. Chang, B. S. Kim, and I. Yoo, "Fourier-Feynman transform, convolution and first variation," Acta Mathematica Hungarica, vol. 100, no. 3, pp. 215-235, 2003.

[19] K. S. Chang, B. S. Kim, and I. Yoo, "Fourier-Feynman transform, convolution and first variation of functionals on abstract Wiener space," Integral Transforms and Special Functions, vol. 10, no. 3-4, pp. 179-200, 2000.

[20] K. S. Chang, T. S. Song, and I. Yoo, "Analytic Fourier-Feynman transform and first variation on abstract Wiener space," Journal of the Korean Mathematical Society, vol. 38, no. 2, pp. 485-501, 2001. 


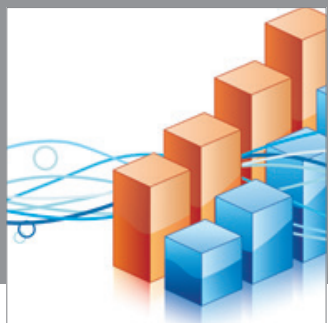

Advances in

Operations Research

mansans

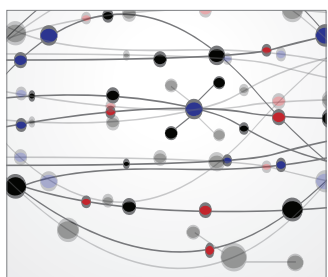

The Scientific World Journal
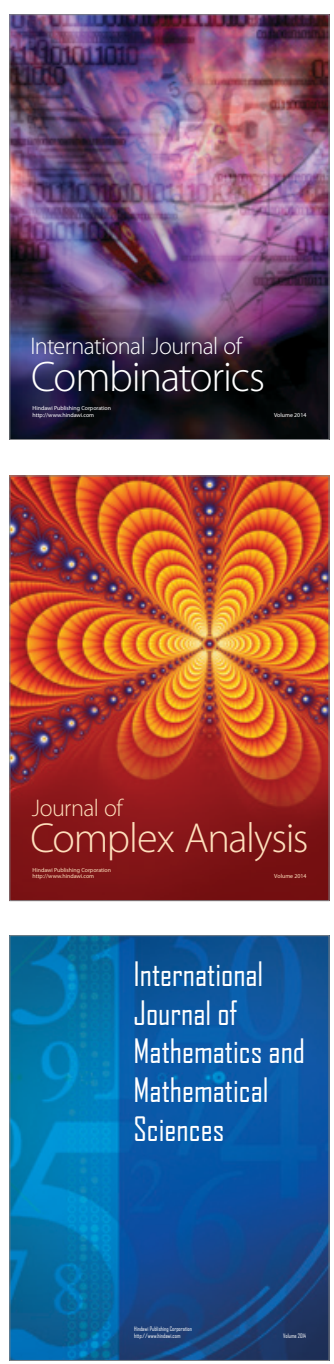
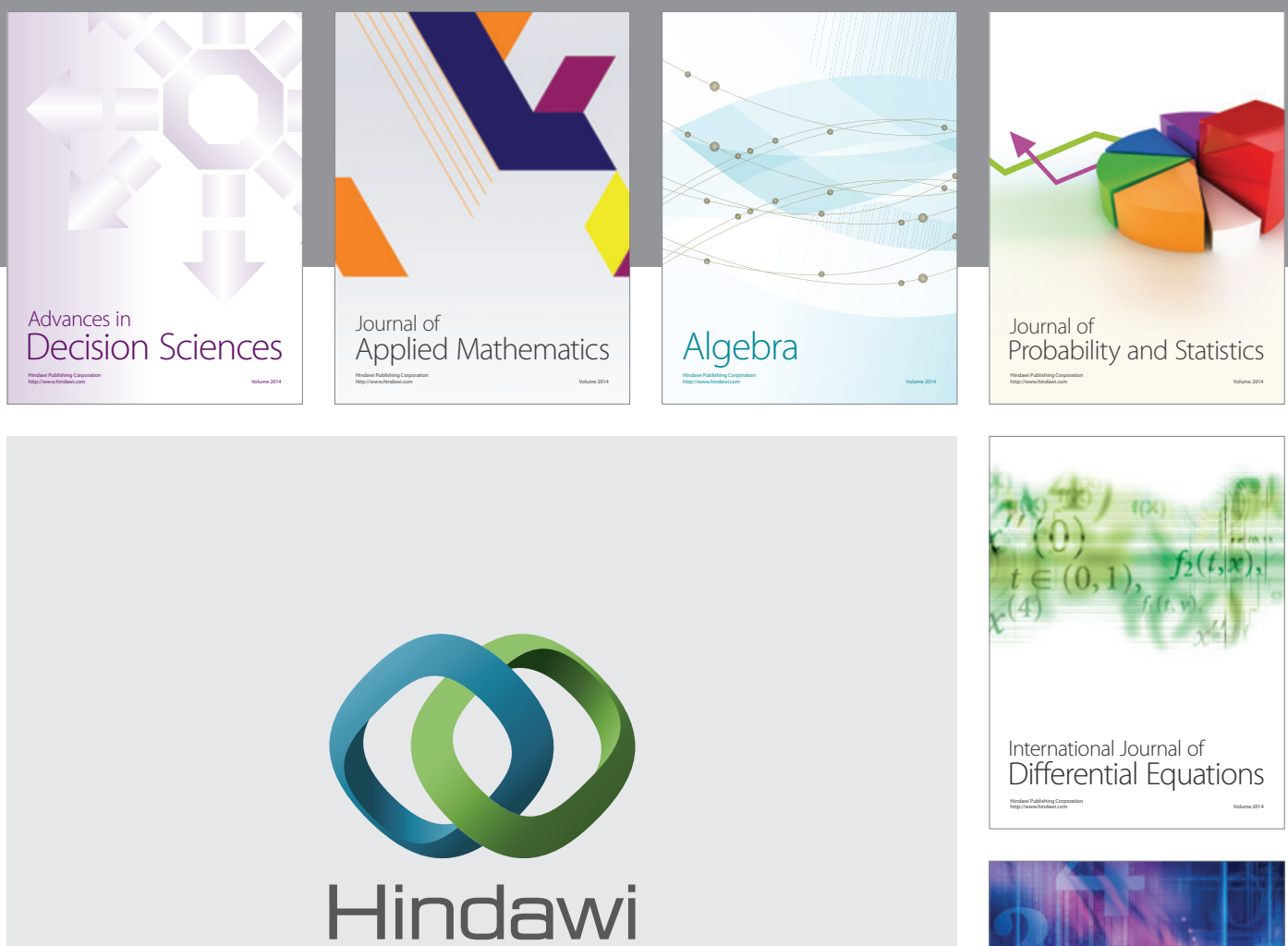

Submit your manuscripts at http://www.hindawi.com
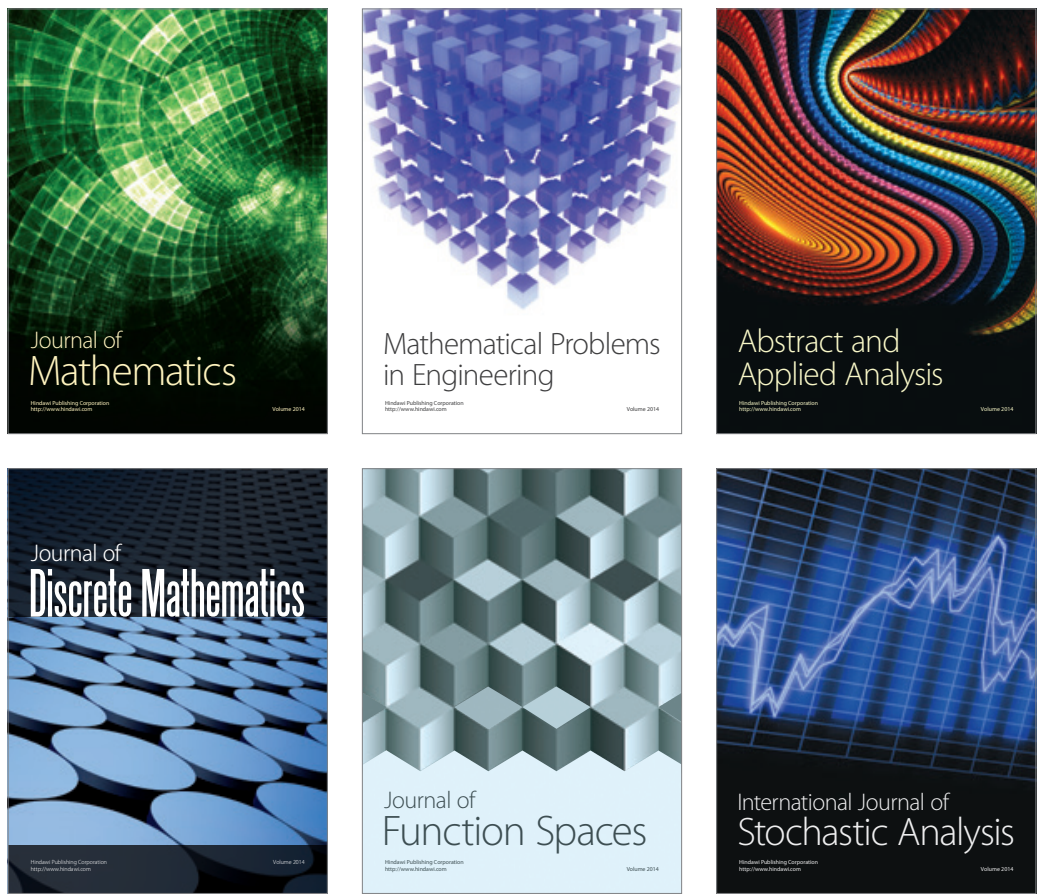

Journal of

Function Spaces

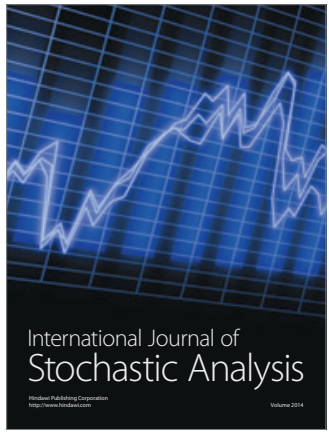

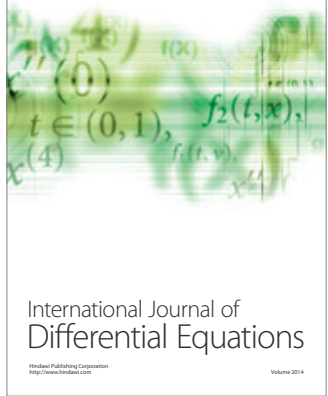
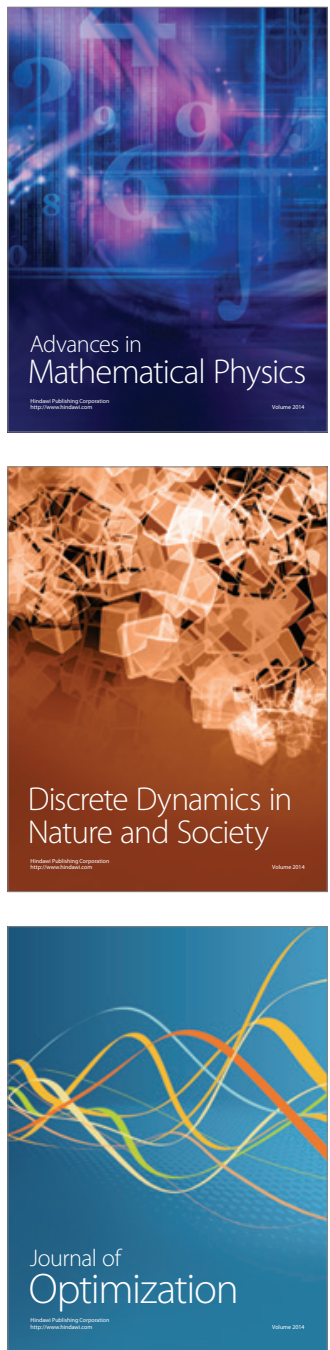